\title{
Digital Preoperative Planning for Total Hip Replacement using Two Dimensional X-ray Imaging
}

\author{
Azrulhizam Shapi'i \\ Industrial Computing \\ Research Group \\ School of Information \\ Technology \\ Universiti Kebangsaan \\ Malaysia
}

\author{
Riza Sulaiman \\ Industrial Computing \\ Research Group \\ School of Information \\ Technology \\ Universiti Kebangsaan \\ Malaysia
}

\author{
Abd Yazid Mohd Kassim \\ Department of Orthopaedic \\ and Traumalogy \\ Medical Center of Universiti \\ Kebangsaan Malaysia \\ Cheras, Kuala Lumpur \\ Malaysia
}

\begin{abstract}
In total hip replacement (THR) surgery, preoperative planning provides valuable information about the anatomy and appropriate implant size. Currently, surgeons predict the size of the implant that will be needed using an analog or conventional method whereby implant templates are overlaid on the patient's $\mathrm{X}$-ray. In this study, we propose to use a digital, computerized method of templating that we expect will be more accurate than the overlay method. For this purpose, we introduced the OrthoHIPTM software for THR preoperative planning. The OrthoHIPTM consists of four main modules; input, scaling, planning and output. This software enables the surgeon to select a digital implant from the database and then digitally overlay it on the two dimensional (2D) X-ray image. In addition, the surgeon can submit the report to a database management system.
\end{abstract}

\section{General terms}

Digital templating.

\section{Keywords}

Total hip replacement, digital, software, implant, preoperative planning, X-ray.

\section{INTRODUCTION}

The medical field at present has many examples of appropriate innovations in the information and computer technology sector that bring benefits to physicians and patients, and also to medical tools, for the prevention of disease. The field of orthopedics has become increasingly influential as the number of patients diagnosed with osteoporosis increases each year. According to surgeons from the Medical Center of University Kebangsaan Malaysia (PPUKM), conventional or manual preoperative planning is still used in total hip replacement (THR) in order to find a suitable implant for hip patients.

Preoperative templating has proven useful for determining the correct size of implant in total hip replacement surgery [1]. Preoperative planning for total hip replacement is essential for a successful outcome. The ultimate choice of the implant system for THR is often a question of surgeon preference. Preoperative evaluation is crucial in total hip replacement (THR) as it determines the size and position of the optimal implant before surgery. A good accuracy of preoperative evaluation results can help improve surgical procedures, save time and minimize damage to the implant after surgery [2]. Manual implant templating is quite difficult to perform due to X-ray film magnification factors that are constantly changing. In the process of digital templating, the templates and images can be automatically scaled to obtain the appropriate magnification scaling factor. In the conventional method, any scaling error in recording skeletal radiographs before surgery will result in significant errors when determining the appropriate implant size [3].

The digital templating process is a pre-surgical process using a digital template [4]. Preoperative planning with overlying templates has become an indispensable part of modern THR, and numerous methods have been proposed for its implementation [3]. With better surgical techniques, aided by the use of computers and sophisticated equipment, THR surgery will eventually be completed within a single day.

Preparation using computerized planning allows surgeons to choose an implant from the database and match it digitally. Some studies have reviewed the utility of digital templating [5-11]. As digital technology improves and becomes more accessible to the health care industry, digital preoperative planning will be used by an increasing number of medical centres. More practices will become filmless and software will be necessary for successful templating. For this specific purpose, we introduce a digital preoperative planning software program for THR.

\section{RESEARCH BACKGROUND}

Total hip replacement (THR) is one of the most successful and effective procedures performed by orthopedic surgeons. Each year, many patients recover after THR surgery. Nowadays, the usual method used in THR is a combination of methods to either replace or not the acetabular and femoral components. There is a history of studies showing that THR surgery lasts a long time. This section will review hip joint disease and THR. Figure 1 shows the anatomy of the hip joint [12].

Although THR has been frequently used since the 1970s, these procedures have specific indications and contraindications. Patients suffering from hip pain and ambulatory (walking) problems, and patients with hip arthrosis radiography records and those who have failed conservative treatment are good candidates for replacement of the hip joint. Examples of conservative treatment for hip disease include weight loss, the use of non-steroidal anti-inflammatory drugs, a change in activities, intra-articular injections and the use of medical devices. However, patients without such criteria will still be considered for THR, but surgeons and patients should be cautious in determining whether or not the risk of undergoing 
this procedure will potentially improve the function and quality of life that may be generated from THR.

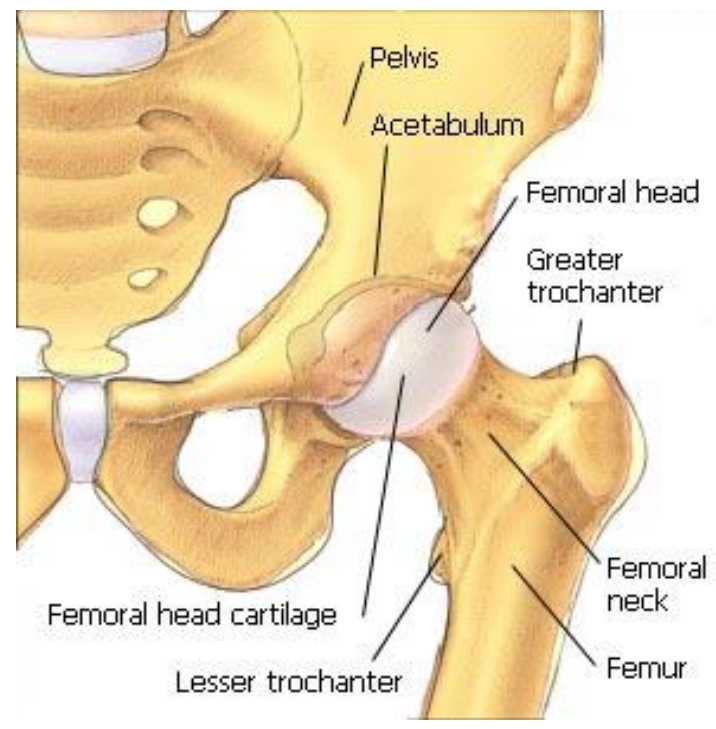

Fig 1: Hip anatomy.

There are many pathological processes that can lead to hip problems, such as rheumatoid arthritis, osteoarthritis, other types of arthritis, post-trauma arthrosis, avascular necrosis (AVN) (see Figure 2), developmental dysplasia of the hip (DDH) (see Figure 3) and dislocation.

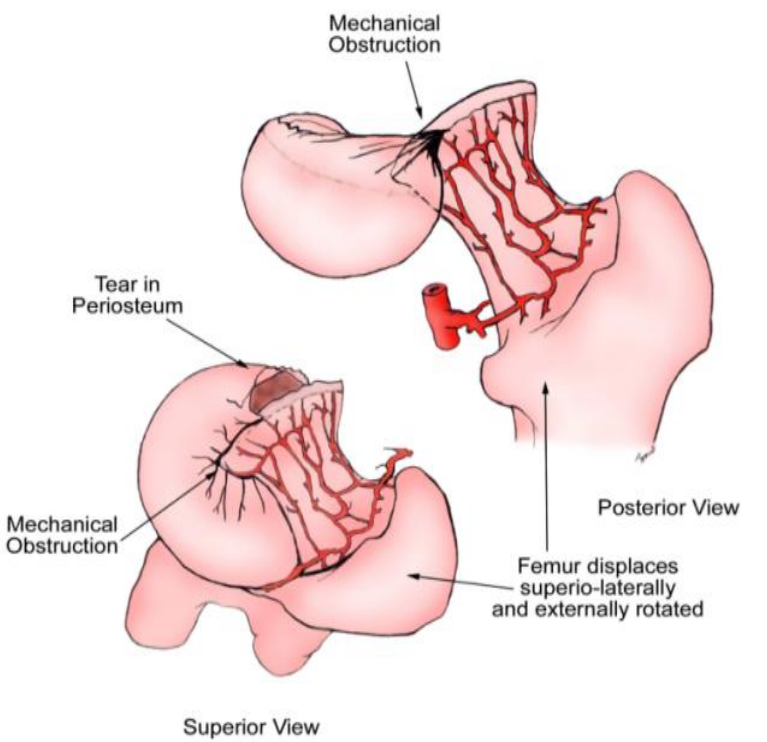

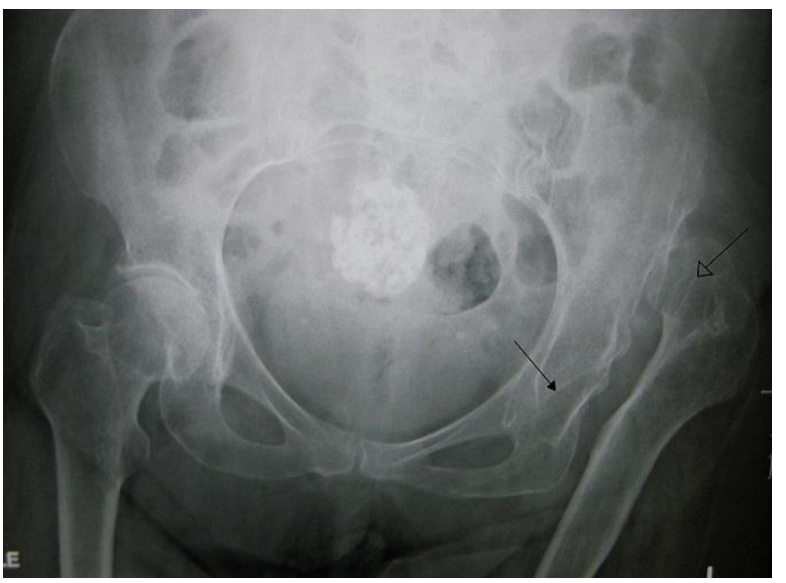

Fig 3: Developmental dsyplasia of the hip (DDH).

Total hip replacement (THR) is a process in which the hip joints are replaced with artificial joints or implants. According to [13], THR is a surgical procedure that replaces the diseased cartilage and joint with artificial materials made of metal and plastic. The use of these implants is intended to help the patients lead a normal life without any interference or pain in the joints when walking and performing daily activities. Anatomically, the hip joint is comprised of a ball and socket joint. The socket is comprised of pelvic bones shaped like a cup, known as the acetabulum, which is connected to the end of the femur, which is shaped like a ball.

The three main components involved in THR are the stem, acetabulum and ball. The corresponding artificial components are designed to ensure that the metal will always rub on the plastic and result in a gentle movement with minimum erosion [3]. In another words, an artificial hip joint (prosthesis) is made up of a ball and socket that fit together to form a joint very similar to patient's hip. Figure 4 shows the implants used in THR [14].
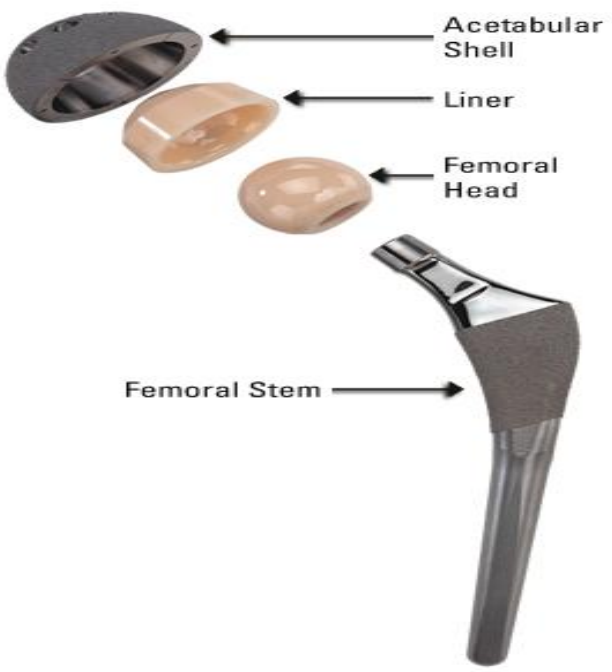

Fig 4: THR implant. 
A hip prosthesis or implant consists of the same basic parts as a human hip joint. The various types of implants are made up of metals or ceramics and plastics. The worn hip socket is replaced by a cup and the worn head of the thigh bone (femur) is replaced by a ball. The ball is attached to a stem that is inserted into the thigh bone for stability. While the prosthesis can restore hip movement, an artificial hip usually has a more limited range of motion than a healthy hip joint. Figure 5 shows the position of the implants after THR is performed [12].

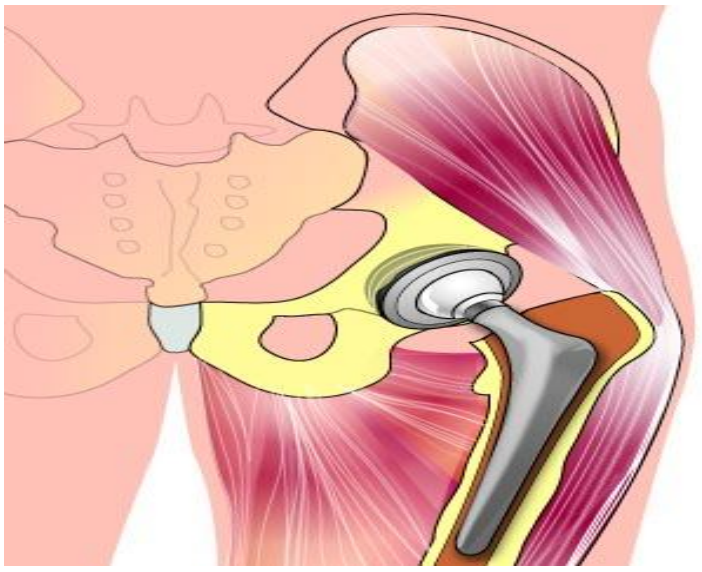

Fig 5: Position of the implants.

\section{MATERIALS AND METHOD}

\subsection{Preoperative Evaluation}

There are several critical components of the preoperative evaluation: a comprehensive history and physical examination including pre-surgical radiographs, and a medical evaluation. Other plans include a sample of pre-surgery radiographs and selection of the approach and surgical techniques. The history and physical examination help to identify whether or not the patient's illness is actually caused by the hip joint, whether or not the hip pain in anyway interferes with the patient's lifestyle and should be considered for THR, and whether or not the patient is a good surgical candidate. Patients with a degenerative hip joint usually complain of pain in the anterior and side of hip, with pain in the groin area that can spread to the anteriorthigh. Some patients also complain of pain in their briskets and knees.

Preoperative radiographs or X-rays are critical parts of preoperative preparation. Radiographs can determine the extent of joint arthrosis and they can also help identify some pathological conditions that require special pre-surgery considerations. Normal preoperative radiographs include an anteroposterior view of the pelvis (see Figure 6). In addition, an anteroposterior view of the proximal femur helps to determine the femoral canal size for pre-surgery implant sizing. The position of the leg view also helps in proximal femoral sizing, and the cross-lateral view is the best for visualizing the acetabulum. In order to determine the optimum implant size for a patient, implant templating on the patient's X-ray image should be implemented.

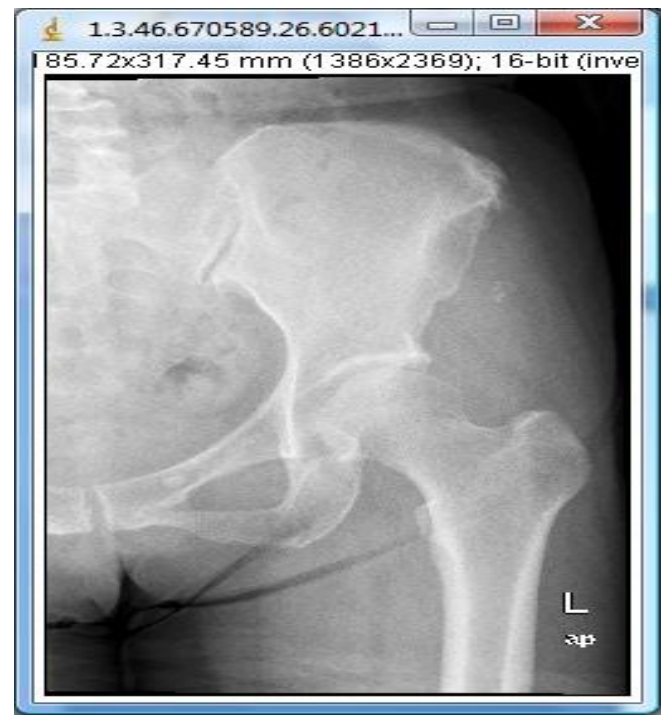

Fig 6: Digital X-ray.

\subsection{Hip Implant Template}

The template refers to standard guidelines used in shaping the frame of the bones. Orthopedic specialists will use this template to match the X-ray image of a patient to an implant so that the optimum size can be determined. Templates are obtained from suppliers in accordance with each prosthetic design. The templates that are available from the Medical Center of University Kebangsaan Malaysia (PPUKM) are Zimmers, with a magnification of $115 \%$ to $120 \%$. These templates are used manually by sticking them onto the AP view radiographs to determine the appropriate size of the stem and acetabulum. Figures 7 and 8 show the implant template used by surgeons in the THR procedure.

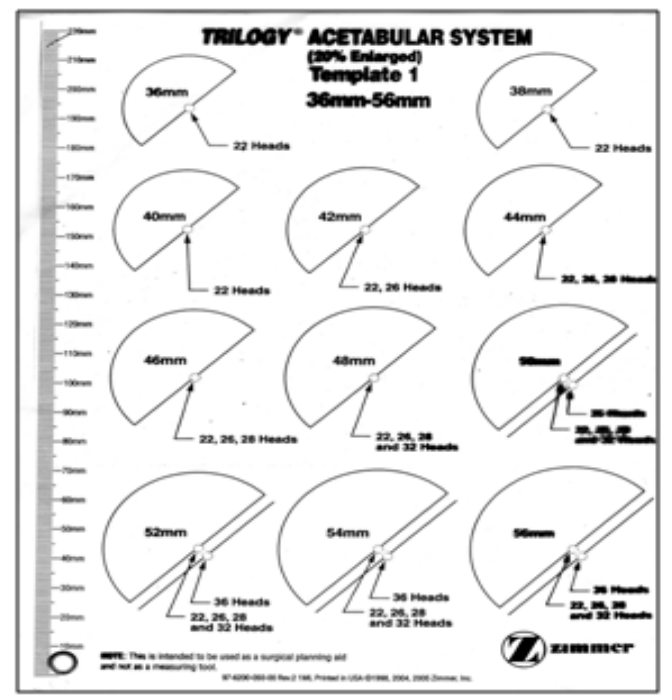

Fig 7: Acetabular template. 


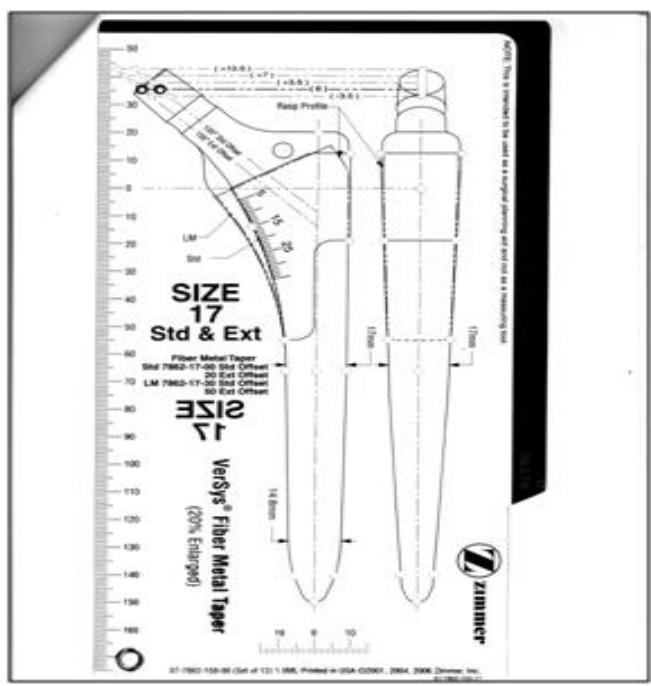

Fig 8: Stem template.

\subsection{Conventional Templating}

Prior to surgery, the template preparation process is very important because it is a screening process in which a surgeon can determine the optimal implant size before surgery. The surgeon will perform the adjustment process of the implant templates first, before bringing the results into the operating room. In the operating room, the surgeon will make the final selection of the optimal implant size. The use of implants in the template customization process on the X-ray image of a patient before surgery can provide important information to surgeons for determining the size of the implant to be used [16]. By using the manual method, any error in template transformation, such as in rotating and scaling while recording the patient's hip bone radiographs prior to surgery, will lead to errors in determining the size of the implant templates. Thus, the templating process should be done cautiously. Figures 9 and 10 show the process of manual implant templating [8].

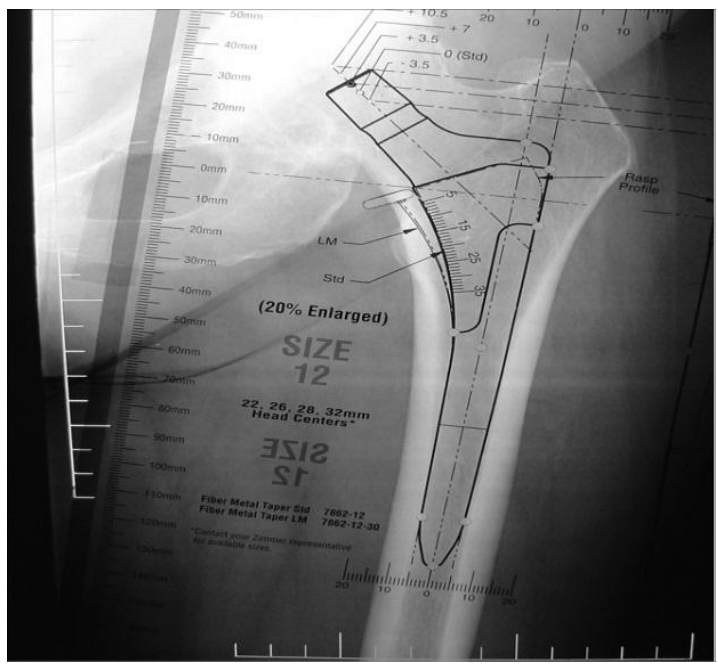

Fig 9: Manual stem templating.

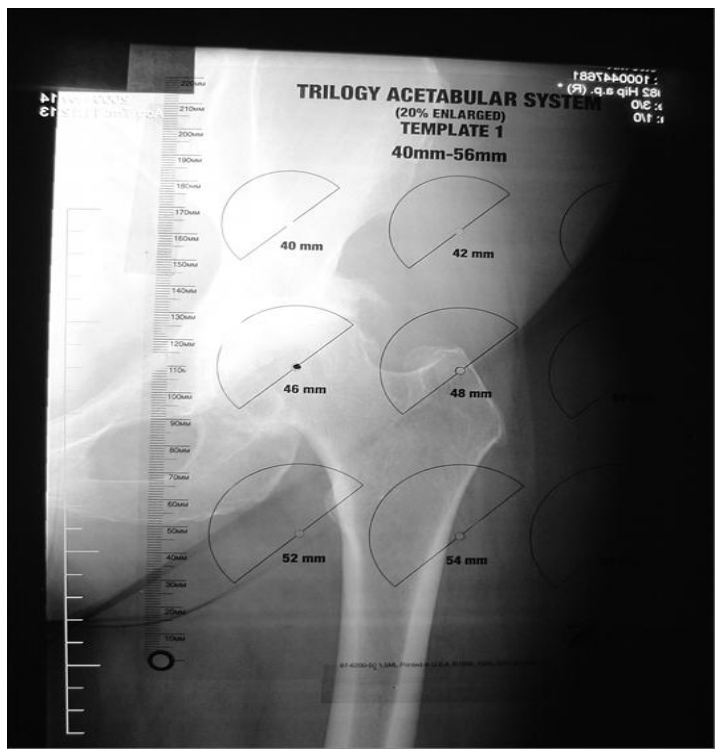

Fig. 10: Manual acetabular templating.

\section{ORTHOHIPTM SOFTWARE}

With the advent of digital X-ray, software has been developed that incorporates the templates into a program which enables the surgeon to measure, without radiographs, the size of the intended femoral and acetabular components. The OrthoHIPтм software was developed in Netbeans in the Java programming language by the Industrial Computing Research Group (ICOMP) researchers at the School of Information Technology, Faculty of Information Science and Technology, University Kebangsaan Malaysia. This software consists of four modules; input, scaling, planning and output. This new software was designed for THR preoperative planning and enables the digitalization of the Zimmer template. Figure 11 shows the structure of the software.

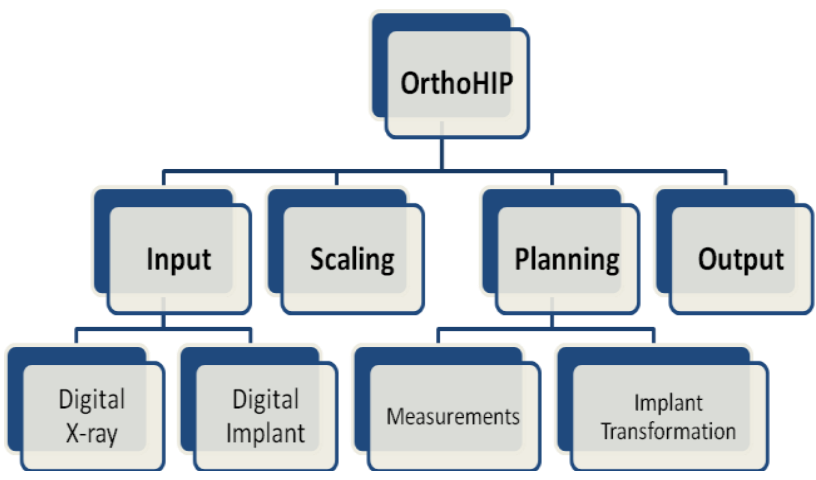

Fig 11: Structure of the OrthoHIP ${ }^{T M}$ software.

\subsection{Input}

Two dimensional (2D) X-ray images are used as the input for the software. Figure 12 shows an example of a 2D digital X-ray used in this study. Hip replacement only involves the AP view (frontal view) of X-ray images. Apart from the images, data contained in the X-ray images will also be used. These data will 
be used in the scaling, planning and output modules [17]. Figure 13 shows the information for the digital X-ray in Figure 12.

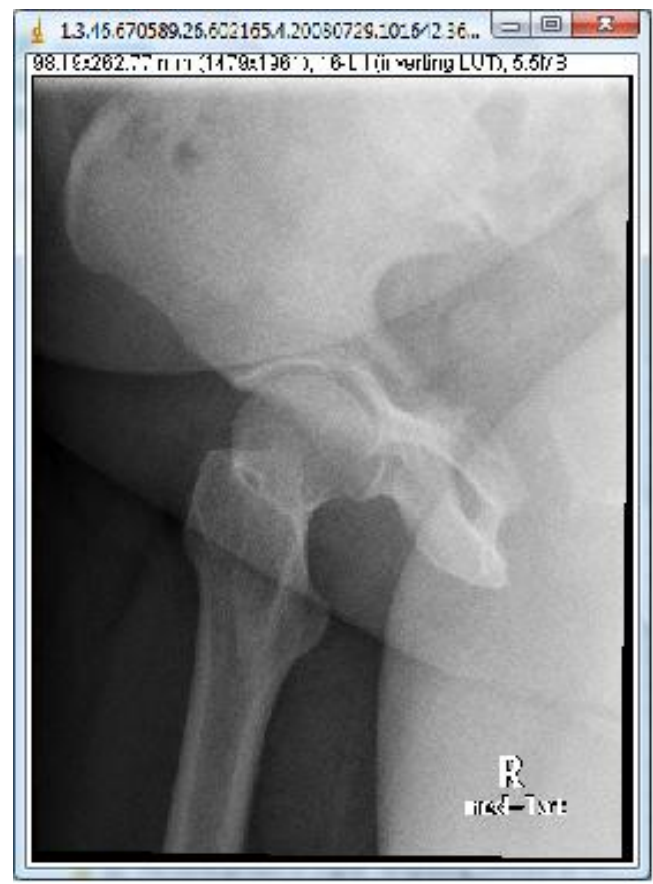

Fig 12: Patient's digital X-ray.

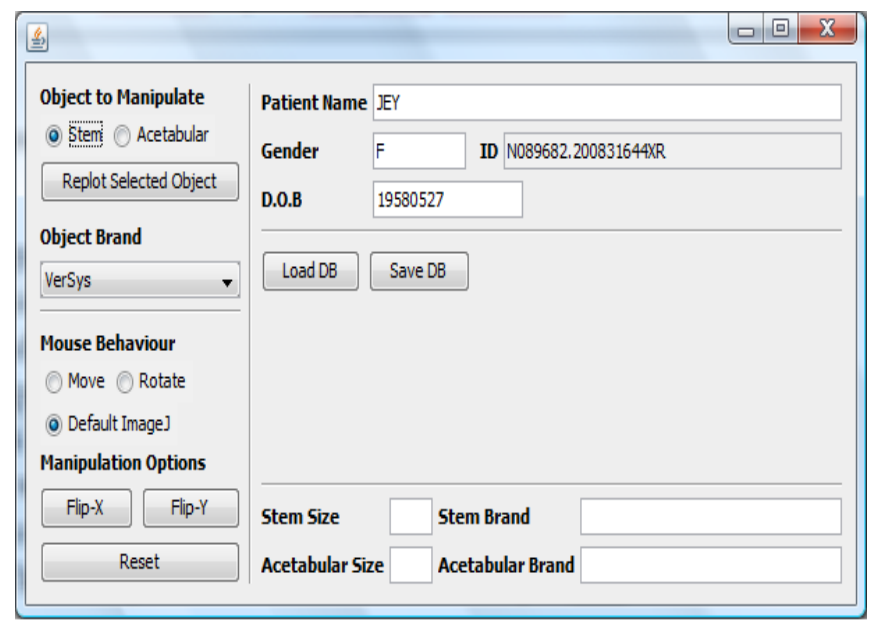

Fig 13: Patient's information.

In this module, a geometric construction of hip joint implant templates will be developed using Autocad 2010 and Adobe Photoshop. Figures 14 and 15 show examples of the digital implants produced.

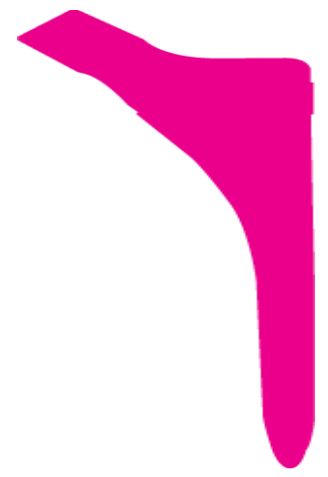

Fig 14: Digital stem implant.

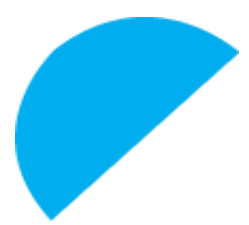

Fig 15: Digital acetabular implant.

\subsection{Scaling and Magnification}

Figure 16 shows the use of digital implants in THR. We can see that the size of the digital implant is not suitable for the X-ray images (the implants are too small). Therefore, we used a scaling method proposed by [18] that can be used to match the implant size with the digital X-ray and computer display. Figure 17 shows the implant size after scaling.

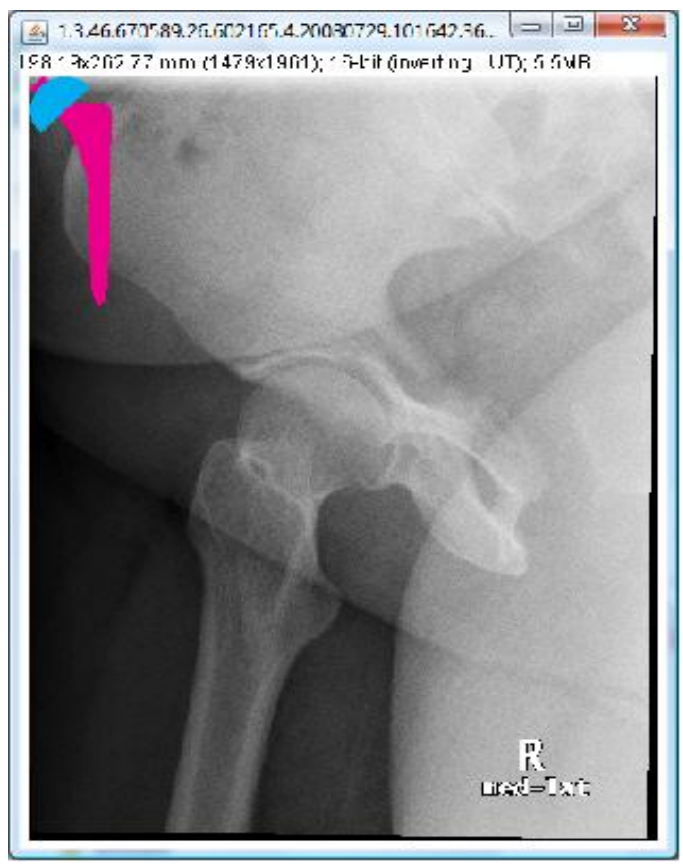

Fig 16: Default implant size without scaling (too small). 


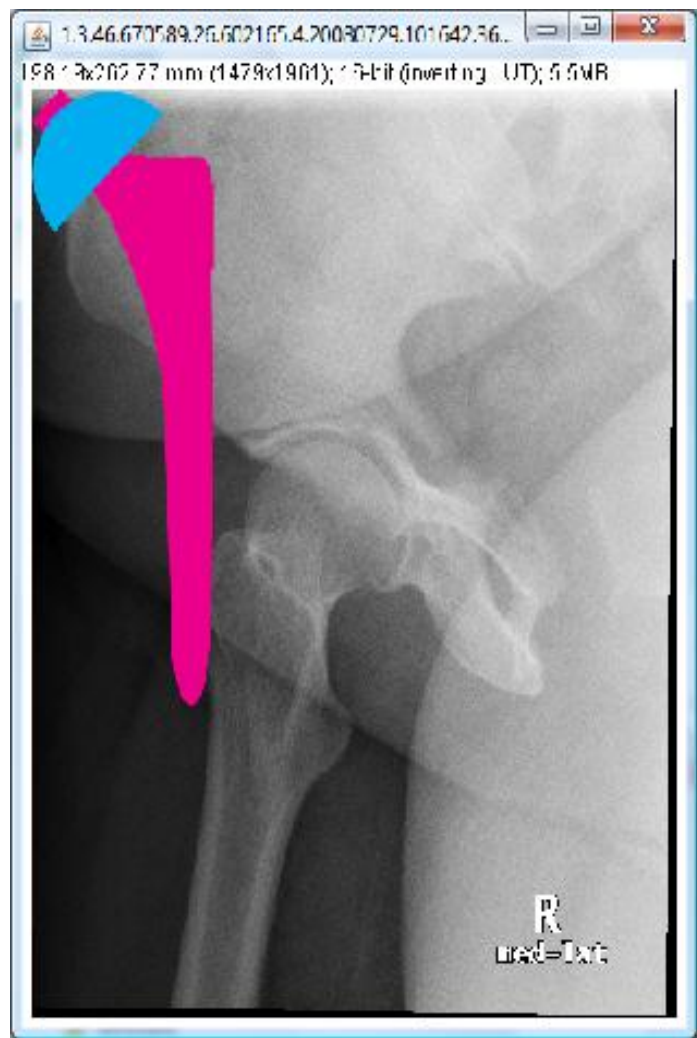

Fig 17: Implant size after the scaling process.

\subsection{Planning}

In this module, the fundamental transformation of the total hip implant on the medical image is performed to determine the optimal implant position. Figure 18 shows the object manipulation technique used in this module [19].

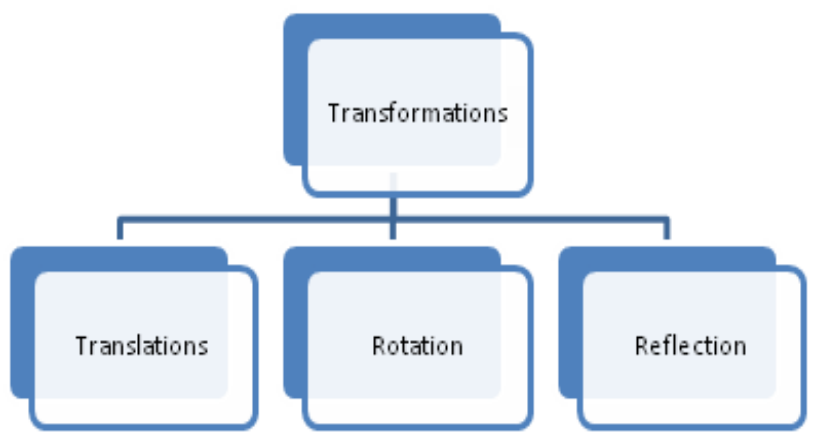

Fig 18: Implant manipulation technique.

Figure 19 shows an implant that has been translated, rotated and reflected (optimal position).

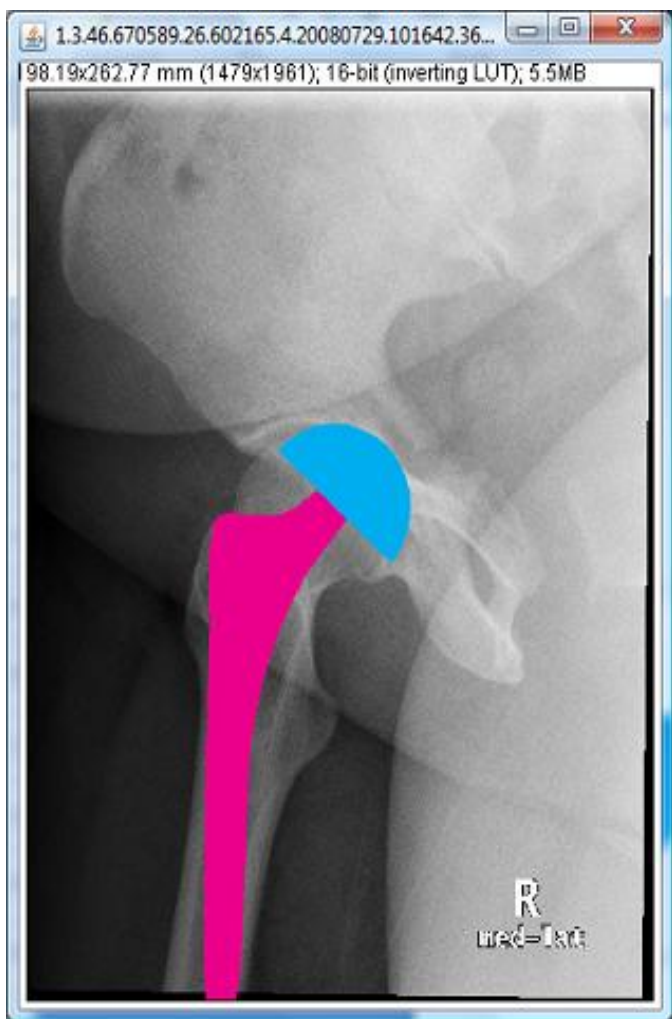

Fig 19: Optimal position for the implant.

\subsection{Output}

In this module, comprehensive planning of a surgical procedure will provide implant specifications as determined by the surgeon as an output from the system (see Figure 20). In addition, there is a facility for producing reports on the specification of these implants. Information can also be stored in the database provided (see Figure 21).

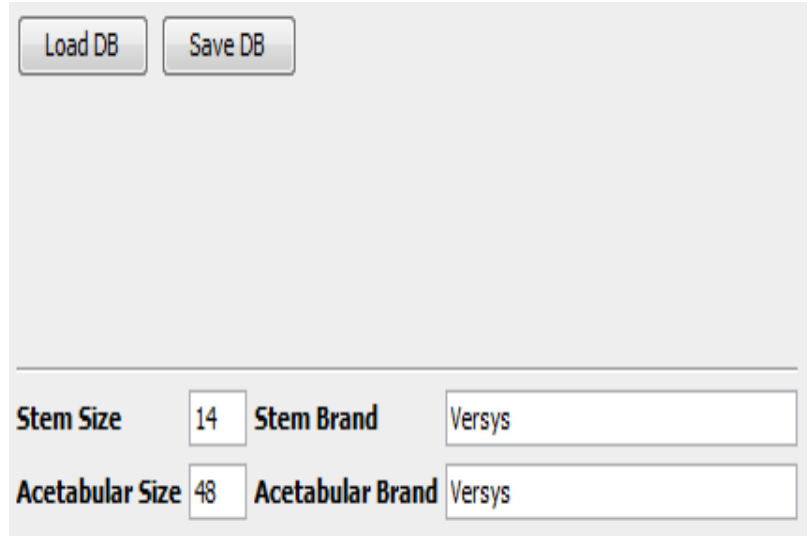

Fig 20: Implant information. 


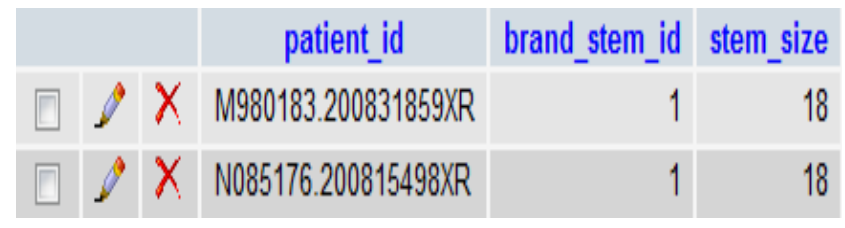

Fig 21: MySQL database for the stem implant.

\section{CONCLUSIONS}

Currently, digital and computer technologies with digital outputs are improving slowly but steadily in the medical center. Preoperative templating has proven useful in determining the optimum size of the prosthesis to be used in total hip replacement (THR). With classical tracing paper now obsolete, we have developed the OrthoHIPTM software to undertake the templating procedure with digital implants and X-ray images. OrthoHIPTM offers a simple solution to the problem of using conventional methods in THR. The problem is solved by the digitalization of implant templates for use in the software program. The software program allows users to choose the implant on a computer prior to surgery. The newly proposed software provides a user-friendly and accurate computer program for surgical planning. This software provides several advantages for the THR process, for example, X-ray film is no longer necessary so there are no radiographs to store or misplace [10].

The limitations of our study include the relatively small number of patients that were evaluated. For future research, we plan to test the software using the data of 30-60 patients. Further clinical studies are planned to confirm both qualitative value of the software and the quantitative precision of the results.

\section{ACKNOWLEDGMENTS}

This research project was conducted in collaboration with $\mathrm{Dr}$ Abdul Yazid Mohd Kassim and Dr Nor Hazla Haflah from the Department of Orthopaedic and Traumalogy and Dr Hamzaini Abd Hamid from the Department of Radiology, Medical Centre of University Kebangsaan Malaysia. This research was also funded by the University Grants UKM-OUP-ICT-35-179/2010 and UKM-GUP-TMK-07-01-035.

\section{REFERENCES}

[1] Murzic, W.J., Glozman, Z. and Lowe, P. 2005. The Accuracy of Digital (filmless) Templating in Total Hip Replacement. 72nd Annual Meeting of the American Academic of Orthopaedic Surgeons.

[2] J.A. Hendrikus, C. Armand, C.V. Laumen, C.V. and J.A. Mourik, "New Digital Preoperative Planning Method for Total Hip Arthroplasties", Journal of Clin Ortho, 467: 909916,2008.

[3] Siti Fairuz, Y. 2009. Knee Joint Replacement automation templates. Msc Thesis, Universiti Kebangsaan Malaysia.

[4] W. Christian, Q. Henning, J. Xu, H. Hansjoerg, V.K. Marius and S. Guido, "Digital Templating in Total Hip Arthroplasty with the Mayo stem", Journal Orthopaedic and Trauma, 1023-1029, 2008.

[5] Zhao, X., Zhu, Z.A., Zhao J., Li, M.Q., Wang, J. and Yu D.G. 2010. The utility of digital templating in Total Hip
Arthroplasty with Crowetype II and III dysplastic hips. International Orthopaedics (SICOT).

[6] P. Gamble, J. de Beer, D. Petruccelli and M. Winemaker, "The accuracy of digital templating in uncemented total hip arthroplasty", Journal of Arthroplasty, $4: 87-93,2009$.

[7] A. González Della Valle, F. Comba, N. Taveras, and E.A. Salvati, "The utility and precision of analogue and digital preoperative planning for total hip arthroplasty", Journal of Int Orthop, 32: 289-294, 2008.

[8] Y. Kosashvili, N. Shasha, E. Olschewski, O. Safir, L. White, A. Gross and D. Backstein, "Digital versus conventional templating techniques in preoperative planning for total hip arthroplasty", Can J Surgery, 52(1): 6-11, 2009.

[9] Todsaporn, F., Amnach, K. and Mitsuhashi W. 2008. Computer-Aided Pre-Operative planning for Total Hip Replacement by using 2D X-ray images. In Proceedings of SICE Annual Conference, August 20-22, Japan.

[10] Michalikova, M., Bednarcikova, L., Petrik, M., Rasi, R. and Zivcak J. 2010. The Digital Pre-Operative Planning of Total Hip Replacement. In Proceedings of 8th IEEE International Symposium on Applied Machine Intelligence and Informatics, January 28-30, Slovakia.

[11] A. Kulkarni, P. Partington, D. Kelly and S. Muller, "Disc Calibration for Digital Templating in Hip Replacement", Journal of Bone and Joint Surgery, 90 (12), 1623-1626.

[12] Hip Surgery website. Current contents http://www.hipsurgery.co.il/, $24^{\text {th }}$ January 2011.

[13] S. Eggli, M. Pisan, M.E. Muller, "The Value of Preoperative Planning for Total Hip Arthroplasty", Journal Bone Jt Surg Br, 80:382-390, 1998.

[14] The Zimmer website. Current contents, http://www.zimmer.com/z/ctl/op/global/, 29th June 2009.

[15] Berry D.J. 2004. Primary Total Hip Arthroplasty. In: Chapman M.W., ed. Chapman's Orthopaedic Surgery.

[16] J. Arora, "The Role of Pre-operative Templating in Primary Total knee Replacement", Knee Surgery Sports Traumatol Arthosc, 187-189, 2004.

[17] Azrulhizam, S., Riza, S., Mohammad, K.H., Abdul, Y.M.K. 2011. Information Retrieval Technique in Medical Imaging Technology. In Proceedings of International Conference of Advanced Science, Engineering and Information Technology, January 14-15, Bangi, Malaysia.

[18] S. Azrulhizam, S. Riza, K.H., Mohammad and M.K., Abdul Yazid and S. Abdullah, "Scaling Technique for Digital Implant in Medical Images Using Pixel Density Algorithm", European Journal of Scientific Research, 47 (1), 24-32. 2010.

[19] S. Azrulhizam, S. Riza, K.H. Mohammad and M.K., Abdul Yazid, "Geometric Transformation Technique for Total Hip Implant in Digital Medical Images", Universal Journal of Computer Science and Engineering Technology, 1(2):79-83, 2010.

[20] ImageJ website. Current contents, http://rsbweb.nih.gov/ij/, 10th October 2010 


\section{AUTHORS PROFILE}

1. Mr Azrulhizam Shapi'i is currently pursuing $P h . D$ in Industrial Computing at School of Information Technology, Universiti Kebangsaan Malaysia. He is working as Lecturer in the School of Information Technology, Faculty of Information Science and Technology, University Kebangsaan Malaysia. His research areas of interest include Computer Aided Design, Medical Imaging, Computer Aided Medical System and Programming.

2. Dr. Riza Sulaiman did his Msc from University of Portmouth, UK and Ph.D from University of Canterbury, New Zealand.
His specializations include Computer Aided Design (CAD), Medical Imaging and Robots Simulation. He is working as Associate Professor in the School of Information Technology, Faculty of Information Science and Technology, University Kebangsaan Malaysia.

3. Dr. Abdul Yazid Mohd Kassim is an orthopaedic surgeon from the Department of Orthopaedic and Traumalogy, Medical Centre of Universiti Kebangsaan Malaysia (PPUKM). 